\title{
1 Vaccine-derived Rotavirus strains in infants in England
}

2

3 Charlotte M. Gower ${ }^{1}$, Jake Dunning, ${ }^{2,3}$ Sameena Nawaz, ${ }^{3}$ David J. Allen ${ }^{3,4}$, Mary E.

4 Ramsay $^{1}$, Shamez N. Ladhani ${ }^{1,5}$

5

$6 \quad{ }^{1}$ Immunisation and Counter-Measures Division, National Infection Service, Public Health 7 England, 61 Colindale Avenue, London, NW9 5EQ, UK

$8{ }^{2}$ Tuberculosis; Acute Respiratory, Gastrointestinal, Emerging and Zoonotic Infections; and 9 Travel and Migrant Health Division (TARGET), National Infection Service, Public Health 10 England, 61 Colindale Avenue, London, NW9 5EQ, UK

$11{ }^{3}$ Enteric Virus Unit, Virus Reference Department, National Infection Service Laboratories, 12 Public Health England, 61 Colindale Avenue, London, NW9 5EQ, UK

$13{ }^{4}$ Department of Pathogen Molecular Biology, London School of Hygiene and Tropical 14 Medicine, Keppel Street, London WC1E 7HT

$15{ }^{5}$ Paediatric Infectious Disease Research Group St. George's University of London, Cranmer 16 Terrace, London SW17 ORE, UK

17 NW9 5EQ, UK. E-mail: shamez.ladhani@phe.gov.uk 


\section{Abstract}

24 Objective: To describe infants with acute gastroenteritis symptoms in primary and secondary 25 care who have the Rotarix ${ }^{\circledR}$ vaccine-derived G1P[8] rotavirus strain identified in their stools.

27 Design: Prospective national surveillance conducted by Public Health England (PHE).

Rotavirus-positive samples from vaccine-eligible children are routinely submitted to PHE for confirmation and general practitioners are requested to complete a surveillance questionnaire

30 for all cases. The modified Vesikari score was used to assess severity of gastroenteritis

31 Setting: England, July 2013 to September 2016

32 Results: 2,637 rotavirus strains were genotyped and $215(8 \%)$ identified as the

33 Rotarix ${ }^{\circledR}$ vaccine-derived G1P[8] strain. There were no Rotarix ${ }^{\circledR}$ vaccine-derived G1P[8]

34 strains detected in unimmunised infants. Rotarix ${ }^{\circledR}$ vaccine-derived G1P[8] strains clustered around the time of rotavirus vaccination and were responsible for $82 \%(107 / 130)$ of rotavirus-positive samples in 2 month-olds and 68\% (36/53) in 3 month-olds. However, 14 samples were obtained more than 7 weeks after the last vaccination date; ten of these specimens were from six children who were subsequently diagnosed with severe combined immune deficiency (SCID). Diarrhoea was the single most common presenting symptom

$40(83.0 \%)$ in infants with Rotarix ${ }^{\circledR}$ vaccine-derived G1P[8] strains, who were also less likely to 41 present with fever, vomiting, dehydration or severe gastroenteritis.

\section{Conclusions}

43 Rotavirus identified in stools of infants around the time of their routine immunisations is 44 most likely be the Rotarix ${ }^{\circledR}$ vaccine-derived G1P[8] strain. Infants with undiagnosed SCID at the time of rotavirus immunisation may experience prolonged gastroenteritis symptoms. The 
46 majority of infants with vaccine strains in their stools more than 7 weeks after immunisation 47 had SCID.

48 


\section{Introduction}

50 Rotavirus is the most common cause of diarrhoea leading to hospitalisation in young children and is associated with considerable healthcare utilisation (1-3). Prior to routine immunisation, rotavirus gastroenteritis (RVGE) was associated with more than 80,000 primary care consultations (2) and 13,000 hospitalisations in the UK each year among children under 5 years (3). On 01 July 2013, a two-dose, oral live-attenuated monovalent rotavirus vaccine, Rotarix $^{\circledR}$ (GlaxoSmithKline Biologicals, Rixensart, Belgium), was introduced into the UK national infant immunisation programme at 8 and 12 weeks of age. Despite strict age restrictions for administering both the first and the second dose of vaccine, the programme rapidly achieved a very high vaccine uptake of $93 \%$ for the two-dose schedule by 25 weeks of age and was associated with a subsequent $15 \%$ decrease in primary care attendance for childhood acute gastroenteritis (AGE) as well as a 77\% reduction in laboratory-confirmed rotavirus infections and a $26 \%$ decline in all-cause AGE-associated hospitalisations across all age groups $(4,5)$.

63

In England, hospital laboratories commonly employ a number of different rotavirus antigen tests with variable sensitivities and specificities to confirm the diagnosis of rotavirus gastroenteritis in children (6). A few of the larger specialist hospitals use more sensitive enzyme immunoassays and, less commonly, reverse-transcriptase polymerase chain reaction (RT-PCR) to confirm the diagnosis. Because of the variable testing practices, and as part of enhanced national surveillance to monitor the impact, effectiveness and safety of the infant rotavirus immunisation programme, Public Health England (PHE) requested hospital laboratories across England to submit all rotavirus-positive stool samples from children in the vaccine-eligible cohort to the national reference laboratory (Enteric Virus Unit; EVU) for confirmation and molecular characterisation. Surveillance of circulating rotavirus genotypes 
74 before and since vaccine introduction has shown that the incidence of the previously most prevalent strain, G1P[8], on which the vaccine is based, has declined most significantly (7). At the same time, the genotype diversity of the remaining wild type rotavirus strains causing gastroenteritis has increased (7). Laboratory surveillance also identified a substantial proportion of samples as Rotarix ${ }^{\circledR}$ vaccine-derived-G1P[8] strains (7). Here we describe the characteristics of infants with confirmed Rotarix ${ }^{\circledR}$ vaccine-derived-G1P[8] strain identified in their stools following the introduction of the rotavirus vaccine into the national immunisation programme and discuss the implications of our findings for frontline clinicians assessing infants with acute gastroenteritis in primary and secondary care.

\section{Methods}

Hospital laboratories routinely report all clinically significant infections, including rotavirus, electronically to PHE through the second-generation surveillance system (SGSS). PHE conducts enhanced national surveillance of all reported rotavirus cases in the vaccine-eligible cohort in England. As part of enhanced surveillance, a questionnaire is sent to general practitioners (GPs) for each case, requesting the rotavirus immunisation history; between 01 July 2013 and 30 June 2015, the questionnaire also requested information to complete a modified Vesikari score for cases $(8,9)$.

Since the introduction of the rotavirus immunisation programme, all NHS laboratories have also been asked to routinely submit rotavirus-positive stool samples in vaccine-eligible children (i.e. those born since 01 May 2013) to the PHE EVU for confirmation (10-12) and molecular characterisation $(13,14)$. Samples from vaccine-eligible cases reported through SGSS and not submitted to PHE are actively followed-up with the reporting hospital virology department. Unlike hospital laboratories, methodologies used at PHE EVU determines wild- 
type rotavirus genotypes according to binomial classification using the virus VP4 (P) and VP7 (G) sequences (as GxP[x]) and further differentiates G1P[8] type viruses between wild-

101 type and vaccine-derived strains. Rotarix ${ }^{\circledR}$ vaccine-derived-G1P[8] strains were defined

102 either: (1) where the sequences of the VP4 and VP7 encoding genes (segments 4 and 9,

103 respectively) demonstrated highest homology with Rotarix ${ }^{\circledR}$ sequences (accession numbers

104 JX943612 and JX943614, respectively); and/or (2) through detection of the Rotarix ${ }^{\circledR}$ sequence using a previously published and validated qRT-PCR assay, specifically targeting the NSP2 gene (segment 8 ) of the Rotarix ${ }^{\circledR}$ strain (13).

108 For this study, all cases identified with a Rotarix ${ }^{\circledR}$ vaccine-derived-G1P[8] strain between 01 109 July 2013 and 30 September 2016 were included in the analysis. For each case, the interval between the date of sample and the last dose of rotavirus vaccination was used to estimate the

111 duration of shedding of the Rotarix ${ }^{\circledR}$ vaccine-derived-G1P[8] strains. Infants with a shedding

112 interval greater than seven weeks were considered as outliers based on the distribution of the

113 data, and investigated further by requesting additional clinical details and underlying

114 conditions from their GP and, if needed, hospital clinicians. Infants confirmed with

115 Rotarix ${ }^{\circledR}$ vaccine-derived-G1P[8] strains who were reported by their GP as unimmunised and

116 those where the date of sample collection preceded the reported vaccination date were also

117 followed-up to investigate the potential source of the Rotarix ${ }^{\circledR}$ vaccine-derived-G1P[8] strain.

\section{Data Analysis}

120 Data are mainly descriptive. Non-normal data are presented as medians with interquartile 121 ranges and compared using the Mann Whitney U test. Proportions are compared using the 122 chi-squared or Fisher's exact test, as appropriate. 


\section{Ethical Approval}

125 PHE has legal permission, provided by Regulation 3 of the Health Service (Control of Patient

126 Information) Regulations 2002, to process confidential information for national surveillance

127 of communicable diseases.

128 (http://www.legislation.gov.uk/uksi/2002/1438/regulation/3/made). This includes PHE's

129 responsibility to monitor the safety and effectiveness of vaccines, and as such, individual

130 patient consent is not required.

131

\section{Results}

133 During 01 July 2013 and 30 September 2016, 2,637 rotavirus strains were genotyped by PHE

134 EVU and $215(8 \%)$ identified as the Rotarix ${ }^{\circledR}$ vaccine-derived-G1P[8] strains. Of the 215

135 strains, eight were from infants who were not UK residents and were, therefore, not followed-

136 up as part of national surveillance as they were not registered with a GP practice.

137 Investigation of seven other cases initially reported as unimmunised by the GP confirmed that

138 all had in fact received the rotavirus vaccine in the six weeks preceding the sample date. In

139 five additional cases, the sample date was reported to be prior to the date of first vaccination,

140 but subsequent investigation revealed that the vaccination date had been reported in error for

141 three cases and the vaccine had in fact been given prior to the sample date. In the remaining

142 two cases, the reported date of vaccination was for the second dose; both infants had been

143 born prematurely and had received their first dose of rotavirus vaccine in hospital at an

144 unspecified date, but prior to the sample date. There were, therefore, no Rotarix ${ }^{\circledR}$ vaccine-

145 derived-G1P[8] strains isolated from unimmunised infants during the surveillance period,

146 despite previous reports (7).

147 
148 Rotarix ${ }^{\circledR}$ vaccine-derived-G1P[8] strains contributed $32 \%(12 / 37)$ of rotavirus-positive

149 samples in infants aged under 2 months (these infants were immunised at 6-8 weeks; i.e.

150 before they become two months old), $82 \%$ (107/130) in 2 month-olds, $68 \%(36 / 53)$ in 3

151 month-olds, $46 \%$ (11/24) in 4-month-olds, $19 \%$ (5/26) in 5 month-olds and less than $1 \%$ in

152 older infants. There were 158 Rotarix ${ }^{\circledR}$ vaccine-derived-G1P[8] samples detected after a first

153 dose of rotavirus vaccine and before the second dose, with a median of 12 (IQR7-21) days

154 after vaccination (range, 0 days to 96 days). In addition, there were 49 samples with a

155 Rotarix ${ }^{\circledR}$ vaccine-derived-G1P[8] strain detected after the second dose of rotavirus vaccine,

156 with a median of $14(\mathrm{IQR}=6-48)$ days after vaccination (range 2 to 420 days) (Figure 1$)$. The

157 interval between the sample date and vaccination was not significant between the first and

158 second dose of Rotarix ${ }^{\circledR}$ (Mann-Whitney U test $-8290, \mathrm{p}=0.51$ ). In the latter group, 19

159 samples were identified with a Rotarix ${ }^{\circledR}$ vaccine-derived-G1P[8] strain more than seven

160 weeks after the last rotavirus vaccination (Figure 1). Of these, six had an incorrect sample

161 date recorded and were, therefore, re-classified. Ten of the remaining samples were from six

162 children who were subsequently diagnosed with severe combined immune deficiency

163 (SCID); three additional cases with sample dates of 112 days, 71 days and 57 days after

164 vaccination, respectively, were from infants who did not have any reported underlying

165 condition; one was subsequently diagnosed with intestinal obstruction.

166

167 Based on the information in the clinical questionnaire completed by the GP, infants with a

168 Rotarix ${ }^{\circledR}$ vaccine-derived-G1P[8] strain were younger than those with wild-type rotavirus

169 gastroenteritis (Table 1). In the former group, diarrhoea was by far the most prevalent

170 presenting symptom (83.0\%) and 54.5\% (68/127) presented with diarrhoea only. By

171 comparison, although infants with wild-type rotavirus gastroenteritis (due to any circulating

172 strain) nearly always also presented with diarrhoea (94.6\%), other symptoms including fever 
173 (48.4\% vs. $20.2 \%, \mathrm{p}<0.001)$ and vomiting (74.2\% vs. $32.0 \%, \mathrm{p}<0.001)$ were more prevalent

174 when compared to infants with a Rotarix ${ }^{\circledR}$ vaccine-derived-G1P[8] strain. Notably, infants

175 with wild-type rotavirus infection were less likely than those with a Rotarix ${ }^{\circledR}$ vaccine-derived-

176 G1P[8] strain to present with diarrhoea only (without vomiting) (95/407 [23.3\%] vs. 68/127

177 [54.5\%]; $\mathrm{P}<0.001)$. Infants with wild-type rotavirus gastroenteritis were also more likely to

178 be dehydrated $(25.1 \%$ vs. $11.4 \%, \mathrm{P}=0.001)$ and have severe gastroenteritis according to the

179 modified Verikari score $(37.5 \%$ vs. $9.8 \%, \mathrm{P}=0.001)$ compared to those with a

180 Rotarix ${ }^{\circledR}$ vaccine-derived-G1P[8] strain (Table 1).

181

182 Discussion

183 During the first three years of the infant rotavirus immunisation programme in England, one

184 in twelve rotavirus strains detected in stool samples from infants in the vaccine-eligible

185 cohort were Rotarix ${ }^{\circledR}$ vaccine-derived-G1P[8] strains. More than $93 \%$ of samples containing

186 Rotarix $^{\circledR}$ vaccine-derived-G1P[8] virus were found in infants within 7 weeks of their first or

187 second Rotarix ${ }^{\circledR}$ vaccination at 8 and 12 weeks of age. Detection of Rotarix ${ }^{\circledR}$ vaccine-derived-

188 G1P[8] strains in infants older than 5 months of age was associated with an underlying

189 diagnosis of SCID; these infants continued to excrete the vaccine-derived for a long period.

190 Infants with Rotarix ${ }^{\circledR}$ vaccine-derived-G1P[8] strains presented predominantly with diarrhoea

191 and, compared to those with wild-type rotavirus gastroenteritis, were less likely to be

192 dehydrated or have severe gastroenteritis as assessed by the modified Vesikari score.

193

194 Currently available point-of-care (POC) or rapid diagnostic tests for rotavirus do not

195 differentiate between rotavirus strains as they are directed toward an antigen (VP6) common

196 across all group A rotaviruses and/or utilise polyclonal reagents which do not discriminate

197 between virus genotypes. Nucleic-Acid Amplification Test (NAAT)-based approaches are 
capable of distinguishing genotypes, and - in the case of G1P[8] - wild from vaccine-derived strains. At present, commercial kit-based platforms do not offer this distinction as part of the multiplex designs, although laboratories may incorporate the test into any in-house methodologies after appropriate validation. The diagnostic tests commonly used by NHS

202 hospital laboratories do not differentiate between wild-type and Rotarix ${ }^{\circledR}$ vaccine-derived-

203 G1P[8] strains; this has important clinical implications.

\section{Vaccine-strains causing symptoms: clinical implications}

In our cohort, stool samples were submitted from symptomatic infants who were assessed in primary or secondary care because of parental concerns. In clinical trials, reported adverse events associated with Rotarix ${ }^{\circledR}$ vaccines include vomiting and diarrhoea. In a recent integrated analysis of the safety and reactogenicity of Rotarix ${ }^{\circledR}$ among $>100,000$ infants enrolled in 28 phase II and III clinical trials, the rates of any vomiting (17.8\% vs. $17.0 \%)$ or diarrhoea (7.8\% vs. $7.5 \%)$ as well as severe (Grade 3 intensity) vomiting (2.7\% vs. $2.4 \%$ ) or

212 diarrhoea (4.9\% vs. $4.5 \%$ ) was similar among recipients of Rotarix ${ }^{\circledR}$ and the placebo group

213 (15). Of the serious adverse events within 30 days of Rotarix ${ }^{\circledR}$ immunisation, however, gastroenteritis ( $0.27 \%$ vs. $0.39 \%$; relative risk $0.65 ; 95 \% \mathrm{CI}, 0.52-0.82 ; \mathrm{P}=0.0002)$ and severe diarrhoea $(0.03 \%$ vs. $0.06 \%$; relative risk $0.48 ; 95 \% \mathrm{CI}, 0.24-0.94 ; \mathrm{P}=0.03)$ were both significantly less common in vaccinated infants compared to the placebo group.

217 In Japan, where two live attenuated oral rotavirus vaccines (Rotarix ${ }^{\circledR}$ and Rotateq ${ }^{\circledR}$ ) have 218 been used voluntarily since 2011 , analysis of 1,824 stool samples from children at outpatient 219 clinics with acute gastroenteritis identified the Rotarix ${ }^{\circledR}$ vaccine-derived-G1P[8] strain in six of $372(1.6 \%)$ rotavirus-positive samples and no Rotateq ${ }^{\circledR}$ vaccine-derived strains (16). Wildtype rotavirus strains and other pathogens such as norovirus, Escherichia coli and enterovirus were also detected in two and four of the six samples, respectively, that were positive for the 
223 Rotarix ${ }^{\circledR}$ vaccine-derived-G1P[8] strain (16). The authors concluded that the contribution of

224 the vaccine-derived strains to the children's symptoms was unclear, although all six had been

225 vaccinated 2-14 days before sample collection. In another study, diarrhoea post-vaccination

226 was reported in $21 \%(13 / 61)$ of infants admitted to hospital within two weeks of receiving the

227 Rotateq $^{\circledR}$ vaccine (17).

228

229 Given that both vomiting and diarrhoea (especially severe symptoms warranting medical

230 attention) are uncommon adverse events following oral rotavirus vaccination, even when

231 solicited in clinical trials, a key question that remains as to whether the vaccine-derived

232 strains identified in the stool samples of symptomatic infants in this study was responsible for

233 the illness or whether another pathology was involved. Additional assessments to identify the

234 cause of the gastrointestinal symptoms, including identification of other pathogens in the

235 stool sample, may help elucidate the role of the Rotarix ${ }^{\circledR}$ vaccine-derived-G1P[8] strains in

236 such infants. In the meantime, clinicians should be cautious when assessing infants presenting

237 with symptoms of acute gastroenteritis during the period after their rotavirus immunisations

238 (typically, 2-5 months of age). In particular, a rotavirus-positive stool sample in a recently

239 immunised infant should be interpreted with caution unless, for G1[P8] strains, the presence

240 of a Rotarix ${ }^{\circledR}$ vaccine-derived G1[P8] has been discounted, particularly in infants who are

241 severely unwell, as there may be another cause of the illness. Another important

242 consideration regarding oral rotavirus vaccination which has previously been reported is that

243 clinicians should be aware of the small but significant increased risk of intussusception

244 during the first week - and up to three weeks - after rotavirus immunisation, especially after

245 the first dose of Rotarix ${ }^{\circledR}(18)$.

246

247 Prolonged shedding and SCID 
248 Infants with the Rotarix ${ }^{\circledR}$ vaccine-derived-G1P[8] strains identified more than 7 weeks after

249 they were given rotavirus immunisation period often had underlying SCID. Prolonged

250 shedding of the Rotarix ${ }^{\circledR}$ vaccine-derived-G1P[8] strain is well-reported in infants with SCID

251 (19) and vaccination of SCID patients with live rotavirus vaccines, including Rotarix ${ }^{\circledR}$, is

252 contra-indicated. Infants with SCID can be diagnosed early through national newborn

253 screening programmes but this is not universally implemented (20-22), including in England,

254 although a pilot study is being planned. In countries without such a screening programme,

255 infants with prolonged gastrointestinal symptoms after rotavirus vaccination and/or shedding

256 of Rotarix ${ }^{\circledR}$ vaccine-derived strain, particularly more than seven weeks following the most

257 recent immunisation, should be assessed for underlying immune deficiency, especially SCID

258 (20).

259

260 Transmission of vaccine-derived strains in the community

261 Another important finding in our study was the lack of Rotarix ${ }^{\circledR}$ vaccine-derived-G1P[8]

262 strains in the stools of unimmunised, symptomatic children during the first three years of the

263 national immunisation programme. Recent studies in neonatal intensive care units also did

264 not identify any transmission of Rotarix $®$ vaccine-derived-G1P[8] strains from immunised to

265 unimmunised infants (23-25). Transmission of the vaccine strain to unimmunised children

266 has been reported, albeit infrequently, and is not associated with any symptoms in the

267 recipients, which is reassuring (26-28). Evidence of such horizontal transmission events is

268 important because it could help explain the indirect (population) protection afforded by the

269 infant programme to unvaccinated children and adults in England (4) and elsewhere (29).

270

271 Strengths and Limitations 
272 The strength of this study lies in the enhanced national surveillance conducted by PHE that

273 began prior to introduction of the rotavirus vaccine into the national immunisation

274 programme (4). In addition to demonstrating population impact, we were able to monitor

275 changes in circulating rotavirus strains following vaccine introduction (7). One limitation,

276 however, was that sample submission rates from vaccine-eligible infants to PHE was

277 relatively poor at the beginning of the programme but increased rapidly once the hospital

278 laboratories implemented local protocols to prospectively submit positive samples to PHE.

279 Additionally, stool samples were taken at the clinicians' discretion and only from infants

280 whose parents were sufficiently concerned about their child to seek medical attentions. It is

281 also possible that clinicians may be more likely to submit stool samples from immunised

282 infants because they would expect such infants to be protected against rotavirus

283 gastroenteritis. Finally, the information needed to calculate the modified Vesikari score, was

284 poorly completed because the individual parameters of the Vesikari score are not routinely

285 recorded in the clinical records and the surveillance questionnaire was sent to GPs several

286 weeks after the diagnosis was confirmed in the infant.

287

288 Conclusions and Clinical Implications

289 Clinicians should be aware that infants may develop acute gastroenteritis symptoms,

290 especially diarrhoea, and have positive rotavirus stool tests after rotavirus vaccination. SCID

291 remains the major contraindication to rotavirus vaccination; those with prolonged

292 gastrointestinal symptoms and/or rotavirus-positive stools after vaccination should be

293 investigated for underlying immunodeficiency, including SCID. 
297 We are grateful to the microbiologists and local authorities, general practitioners, health

298 protection and environmental health specialists who have contributed data and reports to

299 national surveillance systems and the epidemiologists and information officers who have

300 worked on the national surveillance of intestinal infectious diseases for Centre for Infectious

301 Disease Surveillance and Control and Health Protection Services Colindale. We are grateful

302 to the scientists within the PHE Enteric Virus Unit, who perform the confirmatory and

303 characterisation tests for the national surveillance programme, and also Prof David Brown,

304 for his help in establishing the programme.

305

306

307

308

309

310

What is already known

1. Rotavirus is the most common cause of acute gastroenteritis leading to hospitalisation in young children worldwide

2. The live attenuated oral rotavirus vaccines are highly effective in preventing severe rotavirus gastroenteritis and hospitalisations due to rotavirus gastroenteritis

3. Hospital laboratories generally do not distinguish between wild-type rotavirus strains

\section{What this study adds}

1. After implementation of the rotavirus immunisation programme, $8 \%$ of rotaviruspositive stool samples in vaccine-eligible infants were vaccine strains 
2. Most vaccine strains were found in infants within 7 weeks of their first or second Rotarix $^{\circledR}$ immunisation at 8 and 12 weeks of age.

3. Infants with vaccine strains presented mainly with diarrhoea and were less likely to have fever, vomiting, dehydration or severe gastroenteritis than infants with wild type rotavirus.

4. The majority of infants with vaccine strains in their stools more than 7 weeks after immunisation had Severe Combined Immune Deficiency 
1. Giaquinto C, van Damme P. Age distribution of paediatric rotavirus gastroenteritis cases in Europe: the REVEAL study. Scand J Infect Dis. 2010;42(2):142-7.

2. Tam CC, Rodrigues LC, Viviani L, Dodds JP, Evans MR, Hunter PR, et al. Longitudinal study of infectious intestinal disease in the UK (ID2 study): incidence in the community and presenting to general practice. Gut. 2012;61:69-77.

3. Harris JP, Jit M, Cooper D, Edmunds WJ. Evaluating rotavirus vaccination in England and Wales. Part I. Estimating the burden of disease. Vaccine. 2007;25(20):3962-70.

4. Atchison CJ, Stowe J, Andrews N, Collins S, Allen DJ, Nawaz S, et al. Rapid Declines in Age Group-Specific Rotavirus Infection and Acute Gastroenteritis Among Vaccinated and Unvaccinated Individuals Within 1 Year of Rotavirus Vaccine Introduction in England and Wales. J Infect Dis. 2016;213(2):243-9.

5. Thomas SL, Walker JL, Fenty J, Atkins KE, Elliot AJ, Hughes HE, et al. Impact of the national rotavirus vaccination programme on acute gastroenteritis in England and associated costs averted. Vaccine. 2017;35(4):680-6.

6. Atchison CJ, Lopman BA, Harris CJ, Tam CC, Iturriza Gómara M, Gray J, J. Clinical laboratory practices for the detection of rotavirus in England and Wales: can surveillance based on routine laboratory testing data be used to evaluate the impact of vaccination?

. Euro Surveillance. 2009;21:19127.

7. Hungerford D, Allen DJ, Nawaz S, Collins S, Ladhani S, Vivancos R, et al. Impact of rotavirus vaccination on rotavirus genotype distribution and diversity in England, September 2006 to August 2016. Euro Surveill. in press.

8. Schnadower D, Tarr PI, Gorelick MH, O'Connell K, Roskind CG, Powell EC, et al. Validation of the modified Vesikari score in children with gastroenteritis in 5 US emergency departments. Journal of pediatric gastroenterology and nutrition. 2013;57(4):514-9.

9. Freedman SB, Eltorky M, Gorelick M. Evaluation of a gastroenteritis severity score for use in outpatient settings. Pediatrics. 2010;125(6):e1278-85.

10. Iturriza Gomara M, Wong C, Blome S, Desselberger U, Gray J. Molecular characterization of VP6 genes of human rotavirus isolates: correlation of genogroups with subgroups and evidence of independent segregation. Journal of virology. 2002;76(13):6596-601.

11. Iturriza Gomara M, Simpson R, Perault AM, Redpath C, Lorgelly P, Joshi D, et al. Structured surveillance of infantile gastroenteritis in East Anglia, UK: incidence of infection with common viral gastroenteric pathogens. Epidemiol Infect. 2008;136(1):23-33.

12. Iturriza-Gomara M, Dallman T, Banyai K, Bottiger B, Buesa J, Diedrich S, et al. Rotavirus genotypes co-circulating in Europe between 2006 and 2009 as determined by EuroRotaNet, a panEuropean collaborative strain surveillance network. Epidemiol Infect. 2011;139(6):895-909.

13. Gautam R, Esona MD, Mijatovic-Rustempasic S, Ian Tam K, Gentsch JR, Bowen MD. Real-time RT-PCR assays to differentiate wild-type group $A$ rotavirus strains from Rotarix ((R)) and RotaTeq((R)) vaccine strains in stool samples. Human vaccines \& immunotherapeutics. 2014;10(3):767-77.

14. EuroRotaNet network m. http://www.eurorota.net/docs.php [

15. Buyse $\mathrm{H}$, Vinals $\mathrm{C}$, Karkada $\mathrm{N}$, Han $\mathrm{HH}$. The human rotavirus vaccine Rotarix in infants: an integrated analysis of safety and reactogenicity. Human vaccines $\&$ immunotherapeutics. 2014;10(1):19-24.

16. Kaneko M, Takanashi S, Thongprachum A, Hanaoka N, Fujimoto T, Nagasawa K, et al. Identification of vaccine-derived rotavirus strains in children with acute gastroenteritis in Japan, 2012-2015. PLoS One. 2017;12(9):e0184067. 
17. Donato CM, Ch'ng LS, Boniface KF, Crawford NW, Buttery JP, Lyon M, et al. Identification of strains of RotaTeq rotavirus vaccine in infants with gastroenteritis following routine vaccination. J Infect Dis. 2012;206(3):377-83. 18. Stowe J, Andrews N, Ladhani S, Miller E. The risk of intussusception following monovalent rotavirus vaccination in England: A self-controlled case-series evaluation Ref. No: JVAC-D-16-01124. Vaccine. 2016;34(50):6115. (SCID) and rotavirus vaccination: reports to the Vaccine Adverse Events Reporting System (VAERS). Vaccine. 2010;28(40):6609-12.

20. King JR, Hammarstrom L. Newborn Screening for Primary Immunodeficiency Diseases: History, Current and Future Practice. Journal of clinical immunology. 2018;38(1):56-66. 21. Kwan A, Abraham RS, Currier R, Brower A, Andruszewski K, Abbott JK, et al. Newborn screening for severe combined immunodeficiency in 11 screening programs in the United States. Jama. 2014;312(7):729-38. 22. Chien YH, Yu HH, Lee NC, Ho HC, Kao SM, Lu MY, et al. Newborn screening for severe combined immunodeficiency in Taiwan. Int J Neonatal Screen 2017;3:16. Can Be Performed Without Viral Dissemination in the Neonatal Intensive Care Unit. J Infect Dis. 2018;217(4):589-96.

399 24. Hofstetter AM, Lacombe K, Klein EJ, Jones C, Strelitz B, Jacobson E, et al. Risk of Rotavirus Nosocomial Spread After Inpatient Pentavalent Rotavirus Vaccination. Pediatrics. 2018;141(1). 25. Monk HM, Motsney AJ, Wade KC. Safety of rotavirus vaccine in the NICU. Pediatrics. 2014;133(6):e1555-60.

403 26. Dennehy PH, Brady RC, Halperin SA, Ward RL, Alvey JC, Fischer FH, Jr., et al. Comparative 404 evaluation of safety and immunogenicity of two dosages of an oral live attenuated human rotavirus 405 vaccine. Pediatr Infect Dis J. 2005;24(6):481-8.

406 27. Miura H, Kawamura Y, Sugata K, Koshiyama N, Yoshikawa A, Komoto S, et al. Rotavirus 407 vaccine strain transmission by vaccinated infants in the foster home. Journal of medical virology. 408 2017;89(1):79-84.

409 28. Phua KB, Quak SH, Lee BW, Emmanuel SC, Goh P, Han HH, et al. Evaluation of RIX4414, a 410 live, attenuated rotavirus vaccine, in a randomized, double-blind, placebo-controlled phase 2 trial 411 involving 2464 Singaporean infants. J Infect Dis. 2005;192 Suppl 1:S6-s16.

412 29. Anderson EJ. Rotavirus vaccines: viral shedding and risk of transmission. The Lancet 413 Infectious diseases. 2008;8(10):642-9. 
416 Table 1: Characteristics of cases by rotavirus strain type, England 2013-2015.

417

\begin{tabular}{|l|l|l|l|}
\hline Characteristic & Wild type strains & Vaccine derived & P value \\
\hline Age, weeks & $\mathbf{n = 5 3 6 )}$ & G1P[8] (n=174) & \\
\hline Age, months & $54.4(43.3)$ & $10.9(4.6)$ & $\mathrm{P}<0.001$ \\
\hline & $12.5(10.0)$ & $2.5(1.1)$ & $\mathrm{P}<0.001$ \\
\hline Vomiting & & & \\
\hline Diarrhoea & $305 / 411(74.2 \%)$ & $41 / 128(32.0 \%)$ & $\mathrm{P}<0.001$ \\
\hline Fever & $401 / 424(94.6 \%)$ & $112 / 135(83.0 \%)$ & $\mathrm{P}<0.001$ \\
\hline Dehydration & $180 / 372(48.4 \%)$ & $25 / 124(20.2 \%)$ & $\mathrm{P}<0.001$ \\
\hline Severity $*$ \\
Mild/moderate $(1-10)$ \\
Severe $(\geq 11)$
\end{tabular}

419 *modified Vesikari Score (based on the information provided by the GP in the clinical

420 questionnaire, a modified Vesikari score could be calculated for 128 infants with wild-type

421 rotavirus infection and 41 infants with a Rotarix ${ }^{\circledR}$ vaccine-derived-G1P[8] strain).

422

423

424 
$425 \quad$ Figure 1

426 Percentage of 207 Rotarix ${ }^{\circledR v a c c i n e-d e r i v e d-G 1 P[8] ~ s t r a i n s ~ a n d ~ t h e ~ t i m e ~ s i n c e ~ m o s t ~ r e c e n t ~}$

427 documented vaccination in weeks. Underlying conditions of individuals with time since

428 vaccination exceeding seven weeks are indicated.

429 